\title{
Praktische Konsequenzen für Anbieter, Ärzte und Patienten
}

Peter Miny, Siv Fokstuen für die Schweizerische Gesellschaft für Medizinische Genetik

Interessenverbindungen: keine

Korrespondenzen:

Prof. Dr. med. Peter Miny Medizinische Genetik FMH

Medizinisch-genetische Analytik FAMH

Abteilung Medizinische Genetik Universitäts-Kinderspital beider Base (UKBB) und Departement KlinischBiologische Wissenschaften (DKBW) Postfach

4005 Basel

Tel. 0616856254

Fax 0616856011

peter.miny@unibas.ch

Dr. med. Siv Fokstuen

Medizinische Genetik FMH

Service de Génétique Médicale

Hôpitaux Universitaires de Genève

Centre médical universitaire

Rue Michel-Servet 1

1211 Genf 4

Tel. 0223795696

Fax 0223795228

siv.fokstuen@medecine.unige.ch

\section{Zusammenfassung}

Das neue Gesetz und seine Verordnungen verpflichten Anbieter $\mathrm{zu}$ umfassenden Massnahmen der Qualitätskontrolle und -sicherung, verlangen vom auftraggebenden Arzt eine angemessene Beratung und Respektierung der Patientenautonomie und unterstützen das Interesse betroffener Patienten an einem freien Zugang zu qualitativ hochwertiger medizinisch-genetischer Labordiagnostik. Im Bereich der obligatorischen Krankenversicherung sind Kernforderungen des neuen Gesetzes bislang jedoch nicht realisiert.

Die Schweizerische Gesellschaft für Medizinische Genetik (SGMG) begrüsst das neue Gesetz über genetische Untersuchungen beim Menschen und sieht darin einen wichtigen Schritt, international akzeptierte Standards bei medizinisch-genetischen Dienstleistungen für alle verbindlich festzuschreiben. Dazu zählen vor allem die Wahrung der Menschenwürde, die Verhinderung von Missbräuchen, die Sicherstellung einer bestmöglichen Qualität, die Notwendigkeit einer informierten Zustimmung, das Recht auf Nichtwissen sowie ein Verbot der Diskriminierung aufgrund genetischer Merkmale. Die Schweiz gehört damit zu den ersten Ländern weltweit, in denen gesetzliche Leitplanken für die praktische Umsetzung der rasch fortschreitenden medizinisch-genetischen Erkenntnisse, die Auswirkungen auf alle Bereiche der modernen Medizin haben, etabliert wurden. Als vorbildlich kann die gleichzeitige Einsetzung einer unabhängigen Expertenkommission angesehen werden, die unter anderem die Aufgabe hat, «die wissenschaftliche und praktische Entwicklung der genetischen Untersuchungen zu verfolgen, Empfehlungen dazu abzugeben und Lücken in der Gesetzgebung aufzuzeigen» (Art. 35).

\section{Welche Konsequenzen hat das neue Gesetz?}

Für die Anbieter genetischer Untersuchungen Genetische Untersuchungen werden in der Schweiz mehrheitlich durch fünf universitäre
Institute oder Abteilungen für Medizinische Genetik sowie meist grössere, private allgemeine diagnostische Laboratorien angeboten. Darüber hinaus werden insbesondere seltene genetische Untersuchungen krankheitsspezifisch in weiteren, meist universitären, spezialisierten Laboratorien mit unterschiedlicher Affiliation durchgeführt. Neu ist eine Bewilligung für genetische Untersuchungen erforderlich, für die Gesuche bis zum 30. Juni 2007 zu stellen sind. Für akkreditierte Laboratorien wird sich darüber hinaus wenig ändern. Die in der GUMV spezifizierten Voraussetzungen (Details siehe: www.bag. admin.ch/themen/medizin/00683/02724/index. html?lang=de) dürften heute von zahlreichen, jedoch nicht allen Laboratorien bereits erfüllt werden. Insbesondere für letztere sind grosse administrative Anstrengungen absehbar. Sinnvoll erscheint die Regelung, dass die geplanten Inspektionen durch das BAG durch den Nachweis einer Akkreditierung voll ersetzt werden können.

\section{Für auftraggebende Ärztinnen und Ärzte}

Das neue Gesetz betont die Erfordernis einer angemessenen Information und einer formalen Zustimmung des Patienten, bevor genetische (sowohl zytogenetische als auch molekulargenetische) Untersuchungen veranlasst werden. Mindestens für präsymptomatische, pränatale und solche Untersuchungen, die der Familienplanung dienen, ist eine schriftliche Zustimmung zwingend. In diesen Situationen ist die Aufklärungspflicht weitgehend (GUMG, Art. 14) und die Veranlassung der Untersuchung an eine entsprechende Weiterbildung gebunden. Letztere ist jedoch nicht formal spezifiziert, sondern unterliegt der Verantwortung des individuellen Arztes. Für den auftraggebenden Arzt ergibt sich daraus generell die Verpflichtung, eine angemessene nichtdirektive, fachkundige Beratung entweder selbst durchzuführen oder (insbesondere bei komplexeren Untersuchungen) zu veranlassen und dies ausreichend zu dokumentieren. In der Medizinischen Genetik ist es unter diesem Aspekt oft üblich, die wesentlichen 
Punkte eines Beratungsgesprächs in einem Brief an den Patienten schriftlich zu fixieren.

Molekulargenetische Untersuchungen, die eine FAMH-Qualifikation «medizinisch-genetische Analytik» erfordern, werden heute ohne schriftliche Zustimmung des Patienten oder seines gesetzlichen Vertreters in der Schweiz praktisch nicht mehr durchgeführt. Demgegenüber ist eine solche bei Chromosomenuntersuchungen aus historischen Gründen bis heute unüblich. Das GUMG hingegen macht zu Recht keinen Unterschied zwischen beiden Arten von genetischen Untersuchungen. In Zukunft ist auch für Chromosomenuntersuchungen eine schriftliche Zustimmung des Patienten erforderlich, wenn die im Gesetz genannten Voraussetzungen zutreffen. Dies gilt beispielsweise u.a. auch für die relativ häufigen pränatalen Chromosomenuntersuchungen. Der Terminus Chromosomenuntersuchung wird, nebenbei bemerkt, im Laboralltag von zuweisenden Ärzten nicht selten generisch für alle Arten von genetischen Untersuchungen verwendet. Zur korrekten Definition siehe GUMG Art. 3.

Formulare mit einer Liste der wichtigsten Inhalte eines Aufklärungsgesprächs vor der informierten Zustimmung zu einer genetischen Untersuchung finden sich auf der Website der SGMG www.sgmg.ch/sections/Documents/ Statements/publications.htm). Zusammenfassend erfordert das neue Gesetz vom auftraggebenden Arzt eine vermehrte Berücksichtigung von Aspekten der Patientenautonomie mit erweiterter Aufklärung, formaler Zustimmung und Dokumentation der Abläufe wie sie in vielen anderen Bereichen der Medizin ebenfalls üblich ist.

\section{Für Patientinnen und Patienten}

Das klare Bekenntnis zur Patientenautonomie und -information, hohe Anforderungen an die Qualität der Untersuchungen und deren kontinuierliche Überwachung sowie ein uneingeschränktes Verbot einer Diskriminierung aufgrund genetischer Merkmale («Niemand darf wegen seines Erbguts diskriminiert werden»; GUMG Art. 4) sind die Kernpunkte des neuen Gesetzes aus Patientensicht. Sie bieten einen bestmöglichen Schutz vor missbräuchlicher oder inkompetenter Veranlassung genetischer Untersuchungen oder unzureichenden Befundinterpretationen, zumindest im Gültigkeitsbereich des Gesetzes. Wenig ausrichten lässt sich damit allerdings gegen eine Direktvermarktung genetischer Tests im Internet (sog. direct-to-consumer testing) (www.ashg.org/genetics/ashg/news/DTC_ASHG _Draft.pdf), eine Option, die in Zukunft vermutlich stark zunehmen dürfte.
Obwohl vom Gesetz nicht direkt gefordert, wird in der Praxis vermehrt darauf zu achten sein, das Recht eines Kindes auf informationelle Selbstbestimmung nicht zu verletzen. Insbesondere bei familiären Chromosomenanomalien oder rezessiven monogenen Erkrankungen haben Eltern nicht selten den verständlichen Wunsch, Untersuchungen zum Ausschluss oder zur Bestätigung einer (klinisch nicht bedeutsamen) Überträgerschaft bereits im Kindesalter durchführen zu lassen. Damit würde eine Entscheidung, die eigentlich zu einem späteren Zeitpunkt vom Kind zu treffen wäre, allerdings unwiderruflich vorweggenommen. Dies gilt ebenso für präsymptomische Untersuchungen ohne unmittelbare klinische Konsequenzen.

Im Hinblick auf den Abschluss von Versicherungen ausserhalb des obligatorischen Krankenversicherungssystems bringt das neue Gesetz erstmalig eine weitgehende Rechtssicherheit nach Jahren einer Moratoriumsregelung und viel Ungewissheit.

\section{Das GUMG und nachgeordnete gesetz- liche Regelungen (insbesondere KVG)}

Ein grosser Teil aller genetischen Untersuchungen findet heute im Gültigkeitsbereich des Krankenversicherungsgesetzes statt. Aus der Sicht der SGMG stehen Kernbestimmungen des neuen Gesetzes und seiner Verordnungen, insbesondere das Diskriminierungsverbot (GUMG Art. 4) und die geforderte Orientierung der Untersuchungen am Stand von Wissenschaft und Technik (GUMV Art. 3), in diametralem Gegensatz zur gängigen Praxis, die auf den Bestimmungen der Krankenpflegeleistungsverordnung und der Analysenliste beruht. Die Analysenliste ist eine Positivliste, die dem Territorialitätsprinzip unterliegt. Demzufolge werden nur Untersuchungen erstattet, die in der Liste aufgeführt und in der Schweiz durchgeführt werden. Diese Beschränkung, an deren genereller Sinnhaftigkeit wir nicht zweifeln, ist jedoch für medizinisch-genetische Untersuchungen ungeeignet, da sie diagnostische Leistungen für Betroffene und Familien mit seltenen, schweren erblichen Erkrankungen (z.B. Stoffwechselstörungen, Fehlbildungssyndrome) praktisch aus der obligatorischen Krankenversicherung ausschliesst. Heute können mehr als 2000 seltene genetische Erkrankungen verlässlich molekulargenetisch diagnostiziert werden, von denen nur ein Bruchteil (etwa 10-15\%) in der Analysenliste erscheint. Deren Auswahl ist willkürlich. Die Zahl der molekulargenetisch diagnostizierbaren Erkrankungen nimmt praktisch täglich zu. Ein (zwingend krankheitsspezifischer) Antrag zur Aufnahme 
in die Analysenliste ist für die überwiegende Mehrzahl dieser seltenen bis sehr seltenen Erkrankungen aufgrund des heute erforderlichen administrativen Aufwandes völlig illusorisch. Die zusätzliche Forderung, alle Untersuchungen in der Schweiz durchzuführen, kann unter Qualitäts-, wirtschaftlichen und logistischen Aspekten nur als unsinnig bezeichnet werden.

Auch die Verpflichtung, genetische Untersuchungen nach dem Stand von Wissenschaft und Technik durchzuführen, ist im System der obligatorischen Krankenversicherung in vielen Situationen nicht realisierbar. Das im KVG festgeschriebene Konzept von manifester Erkrankung und Prävention wird den Erfordernissen diagnostischer genetischer Untersuchungen nicht gerecht und verhindert bis heute eine Kostenerstattung beispielsweise für Untersuchungen einer uniparentalen Disomie, Überträgerdiagnostik, Kopplungsuntersuchungen oder $\mathrm{Mu}$ tationsnachweis bei einem Indexpatienten.
Zusammenfassend bleibt festzuhalten, dass wesentliche Forderungen des neuen Gesetzes und seiner Verordnungen für den Bereich der obligatorischen Krankenversicherung noch nicht realisiert sind. Betroffene und ihre Familien werden letztlich aufgrund «ihres Erbgutes» (GUMG Art. 4) von gewissen Leistungen der obligatorischen Krankenversicherung ausgeschlossen. Wir sehen z.B. keine sachlichen Gründe, die Untersuchungskosten bei einer Neurofibromatose zu erstatten, bei einer Tuberösen Sklerose jedoch nicht, wie es gegenwärtig geltendem Recht entspricht. Letztere ist weder in der Analysenliste aufgeführt, noch wird die Untersuchung gegenwärtig in der Schweiz angeboten. Aus der Sicht der SGMG handelt es sich hierbei klar um eine Diskriminierung aufgrund administrativer Unzulänglichkeiten, die so rasch wie möglich korrigiert werden sollte. 